\title{
Two-Tier Cache-Aided Full-Duplex Hybrid Satellite-Terrestrial Communication Networks
}

This paper was downloaded from TechRxiv (https://www.techrxiv.org).

\section{LICENSE}

CC BY 4.0

SUBMISSION DATE / POSTED DATE

$16-08-2021 / 21-10-2021$

\section{CITATION}

Ngo, Quynh; Phan, Tran Khoa; Xiang, Wei; Mahmood, Abdun; Slay, Jill (2021): Two-Tier Cache-Aided FullDuplex Hybrid Satellite-Terrestrial Communication Networks. TechRxiv. Preprint. https://doi.org/10.36227/techrxiv.15172038.v2

$\mathrm{DOI}$

10.36227/techrxiv.15172038.v2 


\section{Two-Tier Cache-Aided Full-Duplex Hybrid Satellite-Terrestrial Communication Networks}

\author{
QUYNH T. NGO \\ KHOA T. PHAN, Member, IEEE \\ WEI XIANG, Member, IEEE \\ ABDUN MAHMOOD, Member, IEEE \\ La Trobe University, VIC 3086, Australia
}

JILL SLAY, Member, IEEE

University of South Australia, Adelaide, Australia

\begin{abstract}
Enabling global Internet access is challenging for cellular-based Internet of Things (IoT) due to the limited range of terrestrial network services. One viable solution is to deploy IoT over satellite systems for coverage extension. However, operating a hybrid satellite-terrestrial network (STN) might incur high satellite bandwidth consumption and excessive service latency. Aiming to reduce the content delivery latency from the Internet-connected gateway to the users, this work proposes a two-tier cache-enabled model with full-duplex transmissions where content caches are deployed at the satellite and ground station. A closed-form solution for the successful delivery probability (SDP) of the files is derived considering the requested content distributions and channel statistics. Then, the SDP performance under common caching policies can be evaluated. The results are also used to optimize cache placement under caching capacity constraints. Numerical results demonstrate the performance improvements of the proposed system over those of single-tier cache-aided and half-duplex transmission systems.
\end{abstract}

Index Terms - caching, full-duplex, half-duplex, satellite IoT, successful delivery probability

\section{Introduction}

Although being around for many years, cellularbased technologies for the Internet of Things (IoT) have not fulfilled the demand of globally connecting everyone and everything. To offer service across all geographic

\footnotetext{
"This work was supported by the La Trobe University Postgraduate Research Scholarship, the La Trobe University Full-Fee Research Scholarship, and the Net Zero Scholarship sponsored by Sonepar/SLS." (Corresponding author: K. T. Phan)

Q. T. Ngo, K. T. Phan, W. Xiang, and A. Mahmood are with the School of Engineering and Mathematical Sciences, La Trobe University, VIC, Australia (email: \{T.Ngo, K.Phan,W.Xiang, A.Mahmood\}@latrobe.edu.au). J. Slay is with the School of Computer and Information Science, University of South Australia, Adelaide, Australia (email: Jill.Slay@unisa.edu.au).
}

0018-9251@ 2020 IEEE regions, integrating satellites into IoT networks has been touted. With better service reliability and coverage, satellite communications can provide more effective solutions for IoT than cellular networks. For instance, satellites can provide more than $99.9 \%$ availability with proper constellation arrangements [1] and can cover an enormous area of the Earth that is suitable for bringing connectivity to rural or remote areas. Reflecting the utilization of satellite systems as a promising solution complimenting terrestrial networks, many projects have been developed such as the new radio supporting integrated satelliteterrestrial networks by 3GPP [2], the Satellite and Terrestrial Network for 5G (SaT5G) [3], SpaceX's Starlink satellite project [4].

Operating a hybrid satellite-terrestrial network (STN), however, poses challenges in service delays and satellite bandwidth costs. A content delivered to end-users from the Internet-connected gateway is relayed through satellite(s) and ground station(s), which extends the serving time in addition to very pricey and often limited satellite bandwidth. Excessive content delivery delay can hinder potential use of STN to support delay-sensitive applications. To address these challenges, caching has been proposed as a mechanism to relocate content storage to edge devices that are closer to users [5]-[12]. In wireless networks, edge caching has been shown to shorten delivery latency and reduce network congestion [13]. In addition, caching contents in satellites or terrestrial stations helps avoid re-transmissions of the same contents and thus significantly reducing in-network traffic [14].

In this paper $^{1}$, we study a two-tier cache-aided STN model considering end-to-end communications (i.e., from the Internet-connected gateway via the satellite and ground station to the end-users). Our work focuses on the content delivery latency analysis in terms of the successful delivery probability (SDP) with full-duplex (FD) transmissions at the satellite and ground station, albeit with imperfect self-interference (SI) cancellation. Deploying FD communications will potentially shorten the service delivery time compared to the half-duplex (HD) mode providing sufficiently effective SI cancellation, and hence increasing the SDP. The main contributions of this paper are summarized as follows:

1) A closed-form SDP expression for the two-tier cache-enabled STN is derived under the FD mode taking into account the requested content distributions, satellite/terrestrial realistic channel statistics, and caching configurations. Three commonly studied caching policies, namely uniform caching, ground station most popular caching, and satellite most popular caching, are also analyzed.

2) Based upon the derived results, the SDP performance of STN under different transmission modes (FD with and without FastForward relaying or HD mode) and

${ }^{1}$ The preliminary results of this work [15] have been presented to the 10th International Conference on Computational Data and Social Networks. 
single-tier cache-aided model can be investigated. The network capacity in terms of the maximum number of supportable users given a minimum SDP requirement is also studied.

3) An SDP maximization-based cache placement is designed subject to the caching capacity constraints of the two cache tiers. While the optimal solution is computationally intractable, low-complexity algorithms are developed to obtain caching policies with superior performance comparing to using common caching configurations.

4) Various simulation scenarios are considered to illustrate the improved SDP performance of the proposed system over its single-tier cache-aided counterparts and HD transmission systems under similar caching constraints and energy consumption.

The remainder of this paper is organized as follows. Section II introduces the related work on edge caching in STN. Section III describes the system model and the transmission scheme for FD transmissions. Section IV analyzes the SDP of the general caching and special caching policies, as well as several baseline schemes such as FD without FastForward relaying protocol, HD transmission scheme, and single-tier cache models. Cache placement design and numerical results are presented in Sections V and VI, respectively. Finally, conclusions are made in Section VII.

\section{Edge caching techniques in STN}

Two types of satellites commonly used in STN are the low earth orbit (LEO) and geosynchronous equatorial orbit (GEO) satellites. Employing LEO satellites can achieve lower transmission latency than using GEO satellites. However, using LEO satellites poses a very challenging problem on dynamic network topology since they are only in contact with stations on the ground for a brief period due to their traveling speeds.

Taking into account the relative motion of LEO satellites and terrestrial users, [5] proposes a cross-time-slot graph for the time-varying topology file distribution in combination with the back-tracing partition directed onpath caching distribution mechanism (BPDM) to reduce the caching overhead. The proposed BPDM focuses on cache location decision using a modified Dijkstra routing algorithm and cache content placement using the popularity-aware multiple regions cooperative cache strategy. Proposing a time-evolving covering set in-network caching mechanism, [6] exploits the local connectivity of different regions in choosing the intermediate nodes with better connectivity that relay the objective file. The proposed mechanism includes a novel event updating graph that captures the time-varying topology of the network, a minimum time-evolving covering set algorithm for cache location decision, and a query-based minimum delay distribution path algorithm for optimal path finding during file access procedure. Leveraging the broadcast characteristic of satellite to efficiently provide traffic offloading for terrestrial networks, [7] proposes an integrated LEO satellite/terrestrial cooperative caching scheme, in which the terrestrial access point is able to share its cached content with the satellite.

Employing GEO satellites in STN is suitable for latency-non-sensitive services due to the satellite high altitude. STN with GEO satellites have the advantage of the static network topology and non-interrupting transmission between the satellites and devices on the ground. Incorporating wireless caching into STN, [8] proposes an amplify-and-forward relaying protocols, where cache is enabled at relays on the ground. The proposal in [8] considers the most popular and uniform content-based cache placement schemes, which shows a substantial improvement in outage probability over the traditional approach without caching. To offload the backhaul of terrestrial networks, [9] proposes using hybrid STN in combination with an off-line edge caching algorithm. Caches are placed at base stations, in which each cache is divided into two parts. The first part is filled with the most local popular files whilst the other part is filled with the most global popular files. On the performance investigation of $5 \mathrm{G}$ edge caching over satellite, [10] uses off-line caching approaches to offload terrestrial backhaul networks. The proposal in [10] focuses on the multimode satellite backhaul in two use cases, i.e., dense urban areas and sparsely populated regions.

It should be emphasized that the system models in [5][10] are single-tier cache-enabled STN where caching is used for terrestrial devices only. Moreover, [8]-[10] consider dual-hop downlink satellite-relay(s)-users transmission only. The satellite uplink communications from the Internet-connected gateway to the satellite is not considered, which is usually the bottleneck in STN due to large bandwidth consumption.

Enabling cache capacity at satellite, [11], [12] propose two-tier caching models, where the first- and secondtier caches are placed in the terrestrial base stations and the satellite, respectively. The two-tier cache system will potentially: (i) reduce service latency since more contents are stored closer to the end-users; (ii) efficiently utilize satellite bandwidth as the contents are prefetched at the satellite during off-peak hours; Furthermore, when same contents are requested by multiple users, having the contents cached at satellite and broadcasting them at once can save satellite bandwidth. The work in [11] focuses on analyzing the satellite bandwidth consumption with an off-line caching scheme where popular contents are cached at each ground station for local services, while the satellite's cache is used for the most popular contents within its coverage (containing multiple ground stations) to take advantage of the satellite's broadcast nature to the ground stations. Non-cached contents can be retrieved from the gateway if needed. On the other hand, our current work focuses on investigating the file delivery time through the successful delivery probability (SDP) metric considering the realistic channel models. SDP analysis is typically relevant in delay-sensitive applications [16]. In addition, while [11] considers a scenario where a 
file is fully cached, our work considers more general caching policies allowing partial caching of files. Hence, caching capability can be exploited better for improved performance. Employing the LEO satellite using a twotier caching model, [12] designs a terrestrial link between the core network and base stations to maintain services when quality satellite connection is absent.

\section{System model}

CONSIDER a STN with a satellite $S$, an Internetconnected satellite gateway $G$, a ground station $G_{s}$, and a set of $K$ ground end-users $U_{i}, i=1, \ldots, K$ as depicted in Fig. 1. In this STN, $S$ is a GEO satellite and $G_{s}$ is a low power ground base station equipped with a satellite receiver. Both $S$ and $G_{s}$ are cache enabled. Assuming there are no direct links from user $U_{i}$ to satellite $S$ and from the ground station to the gateway due to weather factors, long distance, and heavy shadowing.

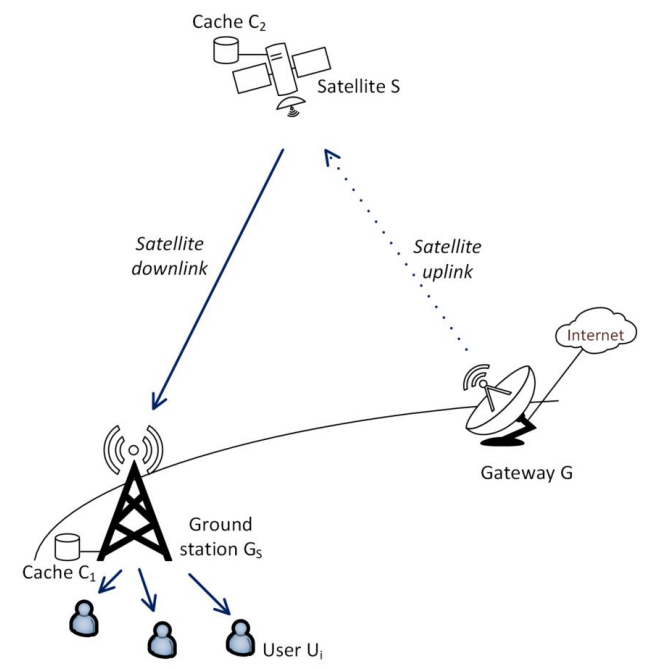

Fig. 1: Satellite IoT networks architecture.

\section{A. Two-tier Caching Model}

Consider a two-tier caching model in which the first tier at $G_{s}$ with storage capacity of $C_{1}$ (bits) and the second tier at $S$ with storage capacity $C_{2}$ (bits). The gateway is connected to the Internet and hosts $N$ files $W_{1}, \ldots, W_{N}$, which are assumed to be of equal size of $F$ (bits) ${ }^{2}$. Without loss of generality, assume $C_{i} / F=$ $N_{i}, i=1,2$ being integer numbers with $N_{1}+N_{2}<N$ as it is not possible to cache all files. The generic caching policy considered in this paper is represented by $\boldsymbol{\mu}_{1}=\left[\mu_{1,1}, \ldots, \mu_{1, N}\right], \quad \boldsymbol{\mu}_{2}=\left[\mu_{2,1}, \ldots, \mu_{2, N}\right]$ where $\mu_{1, n}, \mu_{2, n} \in[0,1]$ are the portions of $W_{n}$ fetched at the first-tier cache (at ground station) and the second-tier cache (at satellite), respectively. It is assumed that the

\footnotetext{
${ }^{2}$ The proposed scheme can be readily extended for unequal file size setting.
}

cached portions of the same file do not have overlapping contents. Other conditions of the caching policy are $\mu_{1, n}+\mu_{2, n} \leq 1, \forall n$ and $\sum_{n=1}^{N} \mu_{i, n}=N_{i}, i=1,2$ as it is optimal to utilize all caching capacities.

\section{B. File Popularity Model}

The request probability $q_{n}$ for file $W_{n}$ follows the Zipf distribution, i.e.,

$$
q_{n}=\frac{n^{-\alpha}}{\sum_{m=1}^{N} m^{-\alpha}}
$$

where $0<\alpha<1$ denotes the Zipf skewness factor [17]. A large value of $\alpha$ means the requests on highpopularity files, whereas a small value of $\alpha$ is related to the requests with heavy-tailed popularity. Without loss of generality, it is assumed that files $W_{1}, \ldots, W_{N}$ have decreasing popularity.

\section{Channel Model}

We consider block-based communications, where a transmission section is accomplished within a coherence time $T$ (seconds). Both large-scale fading and small-scale fading are considered. Large-scale fading is modeled by the distance-dependent power-law path-loss attenuation $d_{m n}^{-\alpha_{i}}$, where $d_{m n}$ denotes the distance between nodes $m$ and $n$, and $\alpha_{i}$ represents the path-loss exponent. For small-scale fading, the channel model proposed in [18] is commonly used for satellite terrestrial communications [8], [19]-[21], while Rayleigh fading channel is commonly used for terrestrial wireless communications.

The (uplink and downlink) satellite-terrestrial links have multipath fading and shadow fading. The shadow fading is composed of the line-of-sight (LOS) shadow fading and multiplicative shadow fading; The multipath fading composes of one LOS and many weak scatter components [18]. Therefore, the channel coefficient $\tilde{h}_{S}(t)$ for the satellite-terrestrial link can be modeled as

$$
\tilde{h}_{S}(t)=A(t) e^{j \vartheta(t)}+Z(t) e^{j \zeta_{0}}
$$

where $j^{2}=-1, A(t)$ is the independent stationary random process representing the amplitude of the scatter components and following the Rayleigh distribution; $Z(t)$ represents the LOS component and follows the Nakagami distribution; $\vartheta(t)$ is the stationary random phase process with a uniform distribution over $[0,2 \pi) ; \zeta_{0}$ is the deterministic phase of the LOS component.

The normalized channel power gain $h_{S}(t)=$ $\left|\tilde{h}_{S}(t)\right|^{2} / \sigma^{2}$, where $\sigma^{2}$ is the additive white Gaussian noise power, has the probability distribution function (PDF) $f_{h_{S}}(x)$ given by [18]

$$
f_{h_{S}}(x)=\alpha_{1} \exp \left(-\frac{x}{b_{1}}\right){ }_{1} F_{1}\left(m_{1}, 1, \sigma_{1} x\right)
$$

with coefficients $\alpha_{1}=\left(\frac{b_{1} m_{1}}{b_{1} m_{1}+\Omega_{1}}\right)^{m_{1}} \frac{1}{b_{1}}, \quad \sigma_{1}=$ $\frac{\Omega_{1}}{b_{1}\left(b_{1} m_{1}+\Omega_{1}\right)}$, where $b_{1}=\mathbb{E}\left[|A(t)|^{2}\right]$ represents the 
average power of the scatter components ; $\Omega_{1}=$ $\mathbb{E}\left[|Z(t)|^{2}\right]$ represents the average power of the LOS component; $m_{1}=\Omega_{1}^{2} / \operatorname{Var}\left[|Z(t)|^{2}\right]$ is the Nakagami parameter; ${ }_{1} F_{1}(., .,$.$) is the confluent hypergeometric$ function of the first kind [22, eq.(9.210.1)]: ${ }_{1} F_{1}(a ; 1 ; z)=$ $\sum_{k=0}^{a-1} \frac{(-1)^{k}(1-a)_{k} z^{k} e^{z}}{(k !)^{2}}$, where $(x)_{n}=\frac{\Gamma(x+n)}{\Gamma(x)}$ denotes the Pochhammer's symbol and $\Gamma(x)$ is Gamma function. Note that we omit the time-dependent index due to the stationarity assumption.

LEMMA 1. The cumulative distribution function (CDF) $F_{h_{S}}(x)$ of $h_{S}(t)$ is

$F_{h_{S}}(x)=\sum_{k=0}^{m_{1}-1} \sum_{q=0}^{k} \frac{\alpha_{1} e^{\left(\sigma_{1}-1 / b_{1}\right) x}(-1)^{2 k-q}\left(1-m_{1}\right)_{k} \sigma_{1}^{k} x^{q}}{k ! q !\left(\sigma_{1}-1 / b_{1}\right)^{k-q+1}}$.

\section{Proof:}

$F_{h_{S}}(x)$ of $h_{S}(t)$ can be derived using elementary functions and finite summations

$$
\begin{aligned}
F_{h_{S}}(x) & =\int_{0}^{x} f_{h_{S}}(y) d y \\
& =\sum_{k=0}^{m_{1}-1} \frac{\alpha_{1}(-1)^{k}\left(1-m_{1}\right)_{k} \sigma_{1}^{k}}{(k !)^{2}} \int_{0}^{x} y^{k} e^{\left(\sigma_{1}-1 / b_{1}\right) y} d y .
\end{aligned}
$$

Using the following result on integration with exponential function:

$$
\int y^{k} e^{c y} d y=e^{c y} \sum_{q=0}^{k} \frac{(-1)^{k-q} k !}{q ! c^{k-q+1}} y^{q},
$$

we can derive (4).

To validate (4), Fig. 2 depicts the PDF and CDF of $h_{S}(t)$ with $\left\{m_{1}, b_{1}, \Omega_{1}\right\}=\{5,0.251,0.279\}$. We can see that as $x$ becomes large, $F_{h_{S}}(x)$ approaches one as expected.

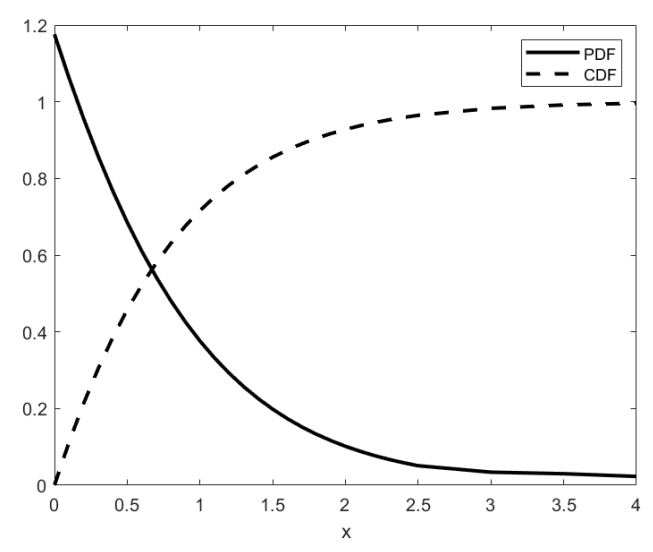

Fig. 2: PDF and CDF of $h_{S}(t)$.

The terrestrial link between the ground station and user $U_{i}$ is modeled as a Rayleigh fading channel with the (normalized) channel power gain $h_{i}(t)$ governed by the following distribution functions

$$
f_{h_{i}}(x)=\frac{1}{\bar{h}_{i}} \exp \left(-\frac{x}{\bar{h}_{i}}\right), \quad F_{h_{i}}(x)=1-\exp \left(-\frac{x}{\bar{h}_{i}}\right)
$$

with $\bar{h}_{i}$ is the average channel power gain.

\section{Full-duplex Transmission with FastForward Relaying}

Assume that the users will be served in a time-division multiple access (TDMA) manner which allows each user to be consecutively active in $T / K$ (seconds). The time slot $T / K$ will be large enough to ensure data freshness in practical IoT applications [23]. Under TDMA, inter-user interference does not exist. Both the satellite and ground station operate in the FD mode. When the channel state information is known at the transmitter, rate adaption is employed and the achievable rates (bps) on the links are

$$
\begin{gathered}
R_{i}=B_{G_{s}} \log _{2}\left(1+P_{G_{s}} h_{i} d_{i}^{-\alpha_{g}}\right), \\
R_{S}=B_{S} \log _{2}\left(1+\frac{P_{S} h_{S} d_{S}^{-\alpha_{s}}}{\beta P_{G_{s}}+1}\right), \\
R_{G}=B_{G} \log _{2}\left(1+\frac{P_{G} h_{G} d_{G}^{-\alpha_{s}}}{\beta P_{S}+1}\right),
\end{gathered}
$$

where $B_{G}, B_{S}$, and $B_{G_{s}}$ are the bandwidths $(\mathrm{Hz})$ of the satellite uplink, downlink, and the terrestrial downlink, respectively; $P_{S}, P_{G}$, and $P_{G_{s}}$ denote the transmit powers of the satellite, gateway, and ground station, respectively; $d_{i}, d_{S}$, and $d_{G}$ are the distances of terrestrial link, satellite downlink and satellite uplink, respectively; and $\alpha_{g}$, and $\alpha_{s}$ represent the path-loss exponents of the terrestrial and satellite links, respectively; $\beta P_{S}$ and $\beta P_{G_{s}}$ represent the normalized residual SI powers at the satellite and ground station respectively, which are assumed to be proportional to the transmit powers with coefficient $\beta \geq 0$ being the SI cancellation quality parameter [24]. For notational simplicity, denote:

$$
\begin{gathered}
\bar{P}_{S} \triangleq P_{S} d_{S}^{-\alpha_{s}} /\left(\beta P_{G_{s}}+1\right), \quad \bar{P}_{G} \triangleq P_{G} d_{G}^{-\alpha_{s}} /\left(\beta P_{S}+1\right), \\
\bar{P}_{G_{s}} \triangleq P_{G_{s}} d_{i}^{-\alpha_{g}} .
\end{gathered}
$$

When a user requests file $W_{n}$, the gateway transfers the non-cached $\left(1-\mu_{1, n}-\mu_{2, n}\right)$ portion to the satellite, which will then send accumulated $\left(1-\mu_{1, n}\right)$ portion to the ground station. By employing the FastForward (FF) protocol [25] with the FD mode at the satellite, the completion time is $\max \left\{\left(1-\mu_{1, n}-\mu_{2, n}\right) F / R_{G},\left(1-\mu_{1, n}\right) F / R_{S}\right\}$. FF protocol allows the relay node to constructively forward the received data with very low processing latency. An example of the delivery scheme using FF protocol is illustrated in Fig. $3^{3}$. Similarly, by deploying the FF protocol with the FD mode at the ground station, the (endto-end) delivery time for transferring the entire file to the

\footnotetext{
${ }^{3}$ We have neglected the propagation delays for simplicity. Otherwise, they can be straightforwardly included in the delivery time expression as approximate constants and the mathematical derivations remain largely unchanged.
} 


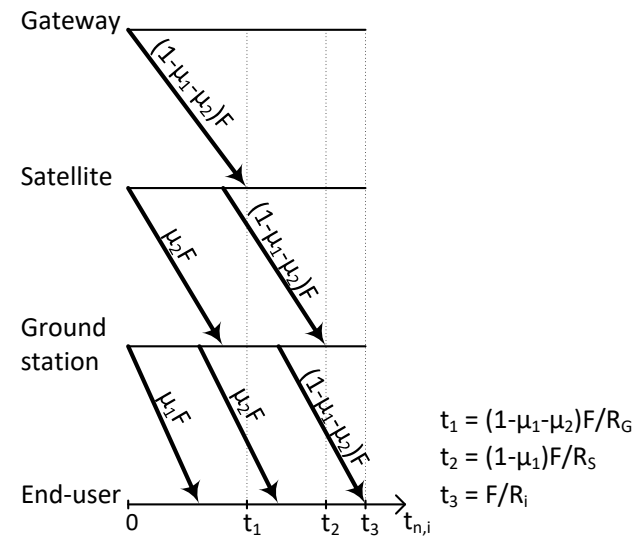

Fig. 3: FD transmissions with FastForward relaying.

user is determined by the time for sending the $\left(1-\mu_{1, n}\right)$ portion over the satellite downlink to the ground station and the time for sending the whole file over the terrestrial link to the user, i.e.,

$t_{n, i}=\max \left\{F / R_{i},\left(1-\mu_{1, n}\right) F / R_{S},\left(1-\mu_{1, n}-\mu_{2, n}\right) F / R_{G}\right\}$

\section{Successful delivery probability analysis}

\section{A. SDP Analysis}

The successful delivery probability (SDP) of serving the requested file $W_{n}$ is the probability that a user receives the file within the user's active time slot. The following analysis is for file $W_{n}$ and user $U_{i}$, and is true for all users.

THEOREM 1. The closed-form expression for the SDP $\psi_{n, i}$ of file $W_{n}$, i.e.,

$$
\psi_{n, i}\left(\mu_{1, n}, \mu_{2, n}\right)=\operatorname{Pr}\left(t_{n, i} \leq T / K\right)
$$

is given in (9).

The probability operator is taken with respect to the channel power gain variables.

Proof:

Substituting (7) into (8) gives rise to

$$
\begin{aligned}
\psi_{n, i} & =\operatorname{Pr}\left(\max \left\{\frac{F}{R_{i}}, \frac{\left(1-\mu_{1, n}\right) F}{R_{S}}, \frac{\left(1-\mu_{1, n}-\mu_{2, n}\right) F}{R_{G}}\right\} \leq \frac{T}{K}\right) \\
& =A_{1} \times A_{2} \times A_{3}
\end{aligned}
$$

where we have

$$
\begin{aligned}
A_{1} & =\operatorname{Pr}\left(F / R_{i} \leq T / K\right) \\
& =1-F_{h_{i}}\left(\left(2^{\tau / B_{G_{s}}}-1\right) / \bar{P}_{G_{s}}\right) \\
A_{2} & =\operatorname{Pr}\left(\left(1-\mu_{1, n}\right) F / R_{S} \leq T / K\right) \\
& =1-F_{h_{S}}\left(\left(2^{\left(1-\mu_{1, n}\right) \tau / B_{S}}-1\right) / \bar{P}_{S}\right) \\
A_{3} & =\operatorname{Pr}\left(\left(1-\mu_{1, n}-\mu_{2, n}\right) F / R_{G} \leq T / K\right) \\
& =1-F_{h_{G}}\left(\left(2^{\left(1-\mu_{1, n}-\mu_{2, n}\right) \tau / B_{G}}-1\right) / \bar{P}_{G}\right)
\end{aligned}
$$

and $\tau \triangleq F /(T / K)$ is the average file serving rate.

We have the following special cases:
- If $W_{n}$ is cached at ground station, we have

$$
\begin{aligned}
\psi_{n, i}(1,0) & =1-F_{h_{i}}\left(\left(2^{\tau / B_{G_{s}}}-1\right) / \bar{P}_{G_{s}}\right) \\
& =\exp \left(\left(2^{\tau_{n} / B_{G_{s}}}-1\right) /\left(\bar{P}_{G_{s}} \bar{h}_{i}\right)\right) .
\end{aligned}
$$

- If $W_{n}$ is cached at the satellite, the SDP is

$$
\begin{aligned}
\psi_{n, i}(0,1) & =\left(1-F_{h_{i}}\left(\left(2^{\tau / B_{G_{s}}}-1\right) / \bar{P}_{G_{s}}\right)\right) \\
& \times\left(1-F_{h_{S}}\left(\left(2^{\tau / B_{S}}-1\right) / \bar{P}_{S}\right)\right) .
\end{aligned}
$$

- If $W_{n}$ is fully retrieved from the gateway, the SDP is

$$
\begin{aligned}
\psi_{n, i}(0,0) & =\left(1-F_{h_{i}}\left(\left(2^{\tau / B_{G_{s}}}-1\right) / \bar{P}_{G_{s}}\right)\right) \\
& \times\left(1-F_{h_{S}}\left(\left(2^{\tau / B_{S}}-1\right) / \bar{P}_{S}\right)\right) \\
& \times\left(1-F_{h_{G}}\left(\left(2^{\tau / B_{G}}-1\right) / \bar{P}_{G}\right)\right) .
\end{aligned}
$$

The SDP of file $W_{n}$ under the effect of $\mu_{1, n}$ and $\mu_{2, n}$ is illustrated in Fig. 4. It shows that the SDP can be improved by deploying the second-tier caching at the satellite, and the larger the caching capacity is, the more SDP improvement is. The SDP is the largest or smallest when the file is cached at the ground station or fully retrieved from the gateway, respectively.

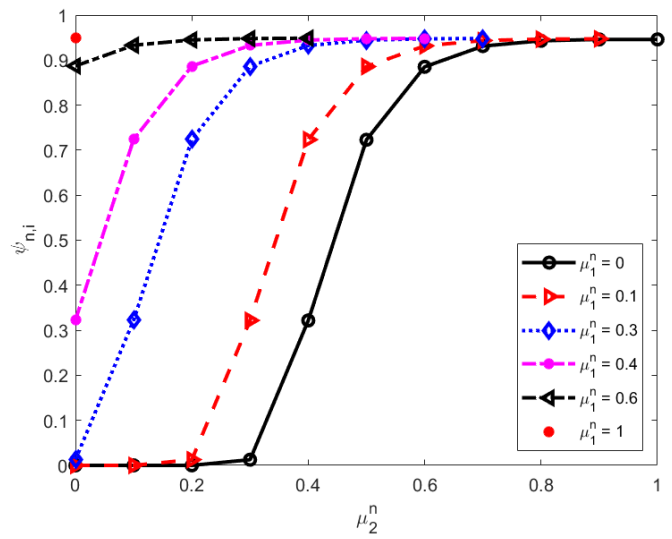

Fig. 4: $\psi_{n, i}$ under different caching portions.

The average SDP of the system is defined as a weighted sum of the users' SDPs:

$$
\begin{aligned}
\psi\left(\boldsymbol{\mu}_{1}, \boldsymbol{\mu}_{2}\right) & =\sum_{i=1}^{K} \omega_{i} \psi_{i}\left(\boldsymbol{\mu}_{1}, \boldsymbol{\mu}_{2}\right) \\
& =\sum_{i=1}^{K} \omega_{i} \sum_{n=1}^{N} q_{n} \psi_{n, i}\left(\mu_{1, n}, \mu_{2, n}\right)
\end{aligned}
$$

with weighting coefficients $\omega_{i} \in(0,1)$ and $\sum_{i} \omega_{i}=1$. In the following, without loss of generality, we assume homogeneous users with $\omega_{i}=1 / K$, and average channel gains $\bar{h}_{i}=\bar{h}_{k}, \forall i, k$. Thus, $\psi=\psi_{i}$.

\section{B. SDP Under Different Caching Policies}

IN this section, we analyze the average SDP under three different caching policies, i.e., uniform caching, 


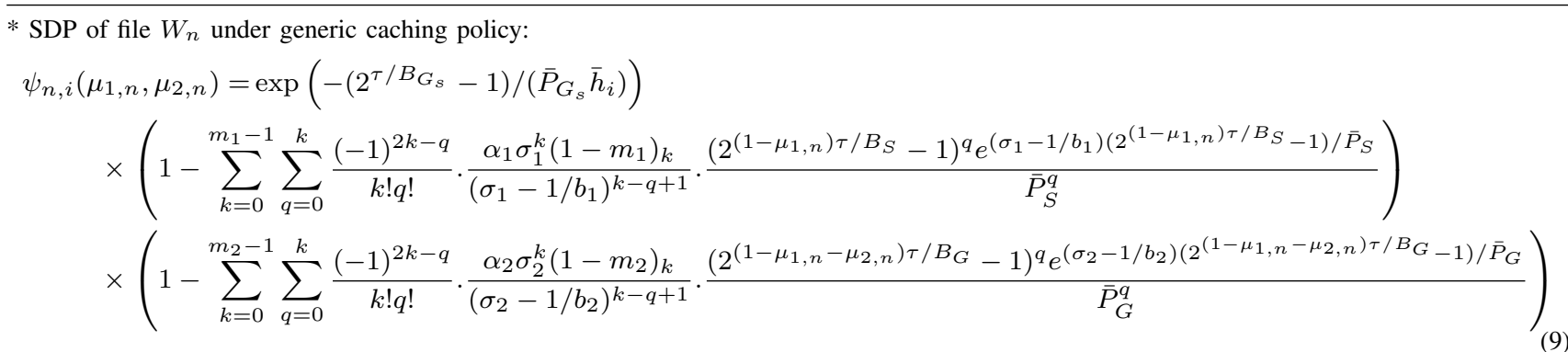

ground station most popular caching, and satellite most popular caching.

\section{Uniform caching policy}

The same portions of $N$ files are cached in both tiers. This caching policy is represented by $\boldsymbol{\mu}_{1}^{\mathrm{u}}=$ $\left[\mu_{1}^{\mathrm{u}}, \ldots, \mu_{1}^{\mathrm{u}}\right], \boldsymbol{\mu}_{2}^{\mathrm{u}}=\left[\mu_{2}^{\mathrm{u}}, \ldots, \mu_{2}^{\mathrm{u}}\right]$ where $\mu_{i}^{\mathrm{u}}=N_{i} / N$. The SDP under this caching policy is

$$
\psi^{\mathrm{u}}=\sum_{n=1}^{N} q_{n} \psi_{n, i}\left(N_{1} / N, N_{2} / N\right) \text {. }
$$

The uniform caching policy does not consider the file popularity and tends to be optimal when all files have similar popularity.

\section{Ground station most popular caching}

The ground station caches $N_{1}$ most popular files, and the satellite caches $N_{2}$ most popular files of the remaining files. This caching policy considers whole file caching and is represented by $\boldsymbol{\mu}_{1}^{\mathrm{g}}=$ $[\underbrace{1, \ldots, 1}_{N_{1}}, 0, \ldots, 0], \quad \boldsymbol{\mu}_{2}^{\mathrm{g}}=[\underbrace{0, \ldots, 0}_{N_{1}}, \underbrace{1, \ldots, 1}_{N_{2}}, 0, \ldots, 0]$. The $\operatorname{SDP} \psi^{\mathrm{g}}$ is $\sum_{n=1}^{N_{1}} q_{n} \psi_{n, i}(1,0)+\sum_{n=N_{1}+1}^{N_{1}+N_{2}} q_{n} \psi_{n, i}(0,1)+$ $\sum_{n=N_{1}+N_{2}+q_{n}}^{N} \psi_{n, i}(0,0)$. This caching policy tends to be better if only a number of files are most popular, i.e., the skewness factor $\alpha$ is large. By caching popular files at the ground station, we can increase the SDP.

\section{Satellite most popular caching}

The satellite caches $N_{2}$ most popular files, and the ground station caches $N_{1}$ most popular files of the remaining files. This caching policy also considers whole file caching and is represented by $\boldsymbol{\mu}_{1}^{\mathrm{s}}=$ $[\underbrace{0, \ldots, 0}_{N_{2}}, \underbrace{1, \ldots, 1}_{N_{1}}, 0, \ldots, 0], \quad \boldsymbol{\mu}_{2}^{\mathrm{s}}=[\underbrace{1, \ldots, 1}_{N_{2}}, 0, \ldots, 0]$. The $\operatorname{SDP} \psi^{\mathrm{s}}$ is $\sum_{n=1}^{N_{2}} q_{n} \psi_{n, i}(0,1)+\sum_{n=N_{2}+1}^{N_{2}+N_{1}} q_{n} \psi_{n, i}(1,0)+$ $\sum_{n=N_{2}+N_{1}+1}^{N} q_{n} \psi_{n, i}(0,0)$. This caching policy is generally more suitable for a two-tier cache-enabled system with multiple ground stations. When most popular files are requested by users associated with different ground stations, the cached files at satellite are broadcast to the ground stations only once. In this case, each ground station does not need to separately cache these popular files. For the system under consideration, we have the following result.
Proposition 1. Assume $N_{1}=N_{2}$. We have $\psi^{\mathrm{g}}>\psi^{\mathrm{s}}$.

Proof:

We have

$$
\begin{gathered}
\psi^{\mathrm{g}}-\psi^{\mathrm{s}}=\sum_{n=1}^{N_{1}} q_{n}\left(\psi_{n, i}(1,0)-\psi_{n, i}(0,1)\right) \\
-\sum_{n=N_{1}+1}^{N_{1}+N_{2}} q_{n}\left(\psi_{n, i}(1,0)-\psi_{n, i}(0,1)\right) \\
=\left(\sum_{n=1}^{N_{1}} q_{n}-\sum_{n=N_{1}+1}^{N_{1}+N_{2}} q_{n}\right)\left(\psi_{n, i}(1,0)-\psi_{n, i}(0,1)\right)>0 .
\end{gathered}
$$

\section{Baselines}

\section{Single-tier caching}

Our derived results can be used to evaluate the SDP of single-tier caching policies. In the single-tier scenario, only cache $C_{1}$ is enabled at the ground station. There are two cases:

- When the same portion of $N$ files are cached at the ground station, we have:

$$
\psi_{\mathrm{s}}^{\mathrm{u}}=\sum_{n=1}^{N} q_{n} \psi_{n, i}\left(N_{1} / N, 0\right)
$$

- When $N_{1}$ most popular files are cached at the ground station, we have:

$$
\psi_{\mathrm{s}}^{\mathrm{g}}=\sum_{n=1}^{N_{1}} q_{n} \psi_{n, i}(1,0)+\sum_{n=N_{1}+1}^{N} q_{n} \psi_{n, i}(0,0) .
$$

\section{Full-duplex without FastForward relaying}

The delivery time of file $W_{n}$ without the FF protocol is completed in three time slots as in Fig. 5. In the first time slot, the ground station transmits $\mu_{1, n} F$ to the user while receiving $\mu_{2, n} F$ from the satellite; the satellite transmits $\mu_{2, n} F$ to the ground station while receiving $\left(1-\mu_{1, n}-\right.$ $\left.\mu_{2, n}\right) F$ from the gateway. In the second time slot, the ground station transmits $\mu_{2, n} F$ to the user while receiving $\left(1-\mu_{1, n}-\mu_{2, n}\right) F$ from the satellite. Finally, in the third time slot, the ground station transmits $\left(1-\mu_{1, n}-\mu_{2, n}\right) F$ 
to the user. The delivery time is given by:

$$
\begin{aligned}
\tilde{t}_{n, i}= & \max \left\{\mu_{1, n} F / R_{i}, \mu_{2, n} F / R_{S},\left(1-\mu_{1, n}-\mu_{2, n}\right) F / R_{G}\right\} \\
& +\max \left\{\mu_{2, n} F / R_{i},\left(1-\mu_{1, n}-\mu_{2, n}\right) F / R_{S}\right\} \\
& +\left(1-\mu_{1, n}-\mu_{2, n}\right) F / R_{i} .
\end{aligned}
$$

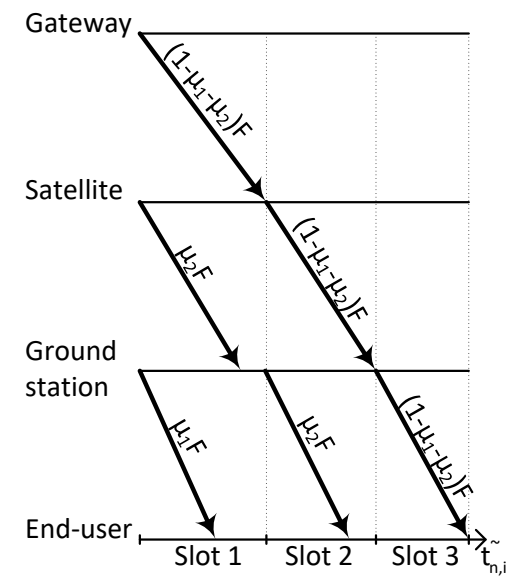

Fig. 5: FD transmissions without FastForward relaying.

The SDP of file $W_{n}$ is $\tilde{\psi}_{n, i}=\operatorname{Pr}\left(\tilde{t}_{n, i} \leq T / K\right)$. We will then evaluate the system SDP $\tilde{\psi}=\sum_{n} q_{n} \tilde{\psi}_{n, i}$ using Monté Carlo simulation in Section VI.

\section{Half-duplex transmission}

In this transmission scheme, both the satellite and ground station adopt the HD mode. The achievable rates on the links are

$$
\begin{aligned}
& R_{i}^{\mathrm{HD}}=B_{G_{s}} \log _{2}\left(1+P_{G_{s}} h_{i} d_{i}^{-\alpha_{g}}\right), \\
& R_{S}^{\mathrm{HD}}=B_{S} \log _{2}\left(1+P_{S} h_{S} d_{S}^{-\alpha_{s}}\right), \\
& R_{G}^{\mathrm{HD}}=B_{G} \log _{2}\left(1+P_{G} h_{G} d_{G}^{-\alpha_{s}}\right) .
\end{aligned}
$$

The timeline for delivering file $W_{n}$ is divided into three time slots as shown in Fig. 6. The delivery time for file $W_{n}$ is

$$
\begin{aligned}
t_{n, i}^{\mathrm{HD}} & =\max \left\{\mu_{1, n} F / R_{i}^{\mathrm{HD}},\left(1-\mu_{1, n}-\mu_{2, n}\right) F / R_{G}^{\mathrm{HD}}\right\} \\
& +\left(1-\mu_{1, n}\right) F / R_{S}^{\mathrm{HD}}+\left(1-\mu_{1, n}\right) F / R_{i}^{\mathrm{HD}}
\end{aligned}
$$

The SDP of file $W_{n}$ is $\psi_{n, i}^{\mathrm{HD}}=\operatorname{Pr}\left(t_{n, i}^{\mathrm{HD}} \leq T / K\right)$. While $\psi^{\mathrm{HD}}=\sum_{n} q_{n} \psi_{n, i}^{\mathrm{HD}}$ is too complex to be found in closed form, it is evaluated using Monté Carlo simulation in Section VI.

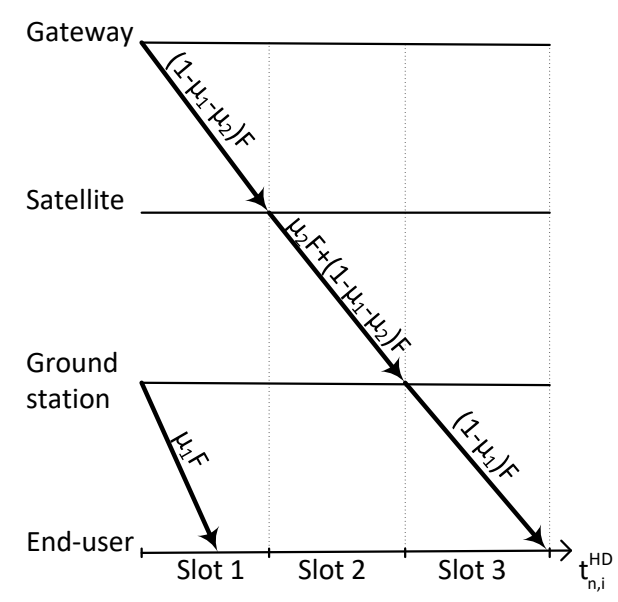

Fig. 6: Half-duplex transmission scheme.

\section{Energy Consumption of FD and HD Transmission}

For a more thorough comparison, we compare the energy consumption of the system in FD/HD modes in addition to the SDP. For the HD transmission scheme, the energies consumption in the three time slots (refer to Fig. 6) are

$$
\begin{aligned}
E_{1}^{\mathrm{HD}} & =\left(P_{G} / \xi_{G}+P_{c, G}\right)\left(1-\mu_{1, n}-\mu_{2, n}\right) F / R_{G}^{\mathrm{HD}} \\
& +\left(P_{G_{s}} / \xi_{G_{s}}+P_{c, G_{s}}\right) \mu_{1, n} F / R_{i}^{\mathrm{HD}} \\
E_{2}^{\mathrm{HD}} & =\left(P_{S} / \xi_{S}+P_{c, S}\right)\left(1-\mu_{1, n}\right) F / R_{S}^{\mathrm{HD}} \\
E_{3}^{\mathrm{HD}} & =\left(P_{G_{s}} / \xi_{G_{s}}+P_{c, G_{s}}\right)\left(1-\mu_{1, n}\right) F / R_{i}^{\mathrm{HD}},
\end{aligned}
$$

with $\xi_{G}, \xi_{S}$, and $\xi_{G_{s}}$ denote the efficiency of the power amplifier at the gateway, satellite and ground station, respectively; and $P_{c, G}, P_{c, S}$, and $P_{c, G_{s}}$ are the constant circuit powers corresponding to the satellite uplink, satellite downlink and terrestrial link, respectively. The total energy consumption under the HD mode is

$$
E^{\mathrm{HD}}=E_{1}^{\mathrm{HD}}+E_{2}^{\mathrm{HD}}+E_{3}^{\mathrm{HD}} .
$$

Different from the HD mode, the FD transmission (with FF relaying protocol) only requires one time slot (refer to Fig. 3) in which all three hops are active. Hence, the total energy consumption is

$$
\begin{aligned}
E^{\mathrm{FD}} & =\left(P_{G} / \xi_{G}+P_{c, G}^{\prime}\right)\left(1-\mu_{1, n}-\mu_{2, n}\right) F / R_{G} \\
& +\left(P_{S} / \xi_{S}+P_{c, S}^{\prime}\right)\left(1-\mu_{1, n}\right) F / R_{S} \\
& +\left(P_{G_{s}} / \xi_{G_{s}}+P_{c, G_{s}}^{\prime}\right) F / R_{i},
\end{aligned}
$$

where $P_{c, j}^{\prime}>P_{c, j}, j \in G, S, G_{s}$ and $\left(P_{c, j}^{\prime}-P_{c, j}\right)$ represents the circuit power to implement self-interference cancellation in the FD system.

\section{Cache placement design}

CACHED contents are placed into caches $C_{1}$ and $C_{2}$ via satellite uplink and downlink transmissions during off-peak hours. In this section, the cached portions $\mu_{1, n}$ and $\mu_{2, n}$ are designed to maximize the SDP. The cache 
placement design problem subject to caching capacity constraints is formulated as follows:

$$
\max _{\boldsymbol{\mu}_{1}, \boldsymbol{\mu}_{2}} \psi\left(\boldsymbol{\mu}_{1}, \boldsymbol{\mu}_{2}\right) \text { s.t. } \sum_{n=1}^{N} \mu_{i, n} \leq N_{i}, i=1,2 .
$$

(13) is a non-linear non-convex optimization, and hence, it is very challenging to find the optimal solution, which can be found using exhaustive search only in general. In the following, we resort to simpler caching placement solutions assuming that the cached files are prefetched with the same portions in each tier. We consider the following two policies.

Generalized uniform caching policy. The caching policy can be described by the vectors $\overline{\boldsymbol{\mu}}_{i}^{\mathrm{u}}=$ $[\underbrace{\bar{\mu}_{i}^{\mathrm{u}}, \ldots, \bar{\mu}_{i}^{\mathrm{u}}}_{\bar{N}}, 0, \ldots, 0]$, where $\bar{N} \leq N$ and $\bar{\mu}_{i}^{\mathrm{u}}=N_{i} / \bar{N}, i=$ 1,2. In this policy, $\bar{N}$ most popular files are being cached with the same portions at the ground station and the satellite. This generalized uniform caching policy is inspired by the fact that uniform caching policy (Section 1) naively caches all files in the library regardless of the file request rate. The cache resource is wasted for prefetching files that are rarely requested. Comparing to the uniform caching policy, this generalized uniform caching policy prioritizes more popular files. This caching policy becomes the uniform caching policy when $\bar{N}=N$.

Generalized ground station most popular caching policy. The same $\bar{\mu}_{1}^{\mathrm{g}}$ portion of $\bar{N}_{1}$ most popular files are cached at ground station, and $\bar{\mu}_{2}^{\mathrm{g}}$ of $\bar{N}_{2}$ most popular files of the remaining files are cached at satellite. This policy can be represented by $\overline{\boldsymbol{\mu}}_{1}^{\mathrm{g}}=[\underbrace{\bar{\mu}_{1}^{\mathrm{g}}, \ldots, \bar{\mu}_{1}^{\mathrm{g}}}_{\bar{N}_{1}}, 0, \ldots, 0]$; and $\bar{\mu}_{2}^{\mathrm{g}}=[\underbrace{0, \ldots, 0}_{\bar{N}_{1}}, \underbrace{\bar{\mu}_{2}^{\mathrm{g}}, \ldots, \bar{\mu}_{2}^{\mathrm{g}}}_{\bar{N}_{2}}, 0, \ldots, 0], \bar{\mu}_{i}^{\mathrm{g}}=N_{i} / \bar{N}_{i}$. Comparing to the whole file caching in the ground station most popular policy (Section 2), the content cached in this policy is partial-file, allowing more popular files being prefetched in the two cache tiers. Hence, the generalized ground station caching policy is more diverse in terms of file popularity. This caching policy boils down to the ground station most popular caching policy when $\bar{N}_{i}=N_{i}, i=1,2$.

We can conduct a simple exhaustive search to find the generalized policy to maximize the SDP in (13), i.e., by searching the number of files $\bar{N}$ or $\bar{N}_{i}$ to be pre-fetched. It can be seen that the obtained policy allows more popular files to be cached closer to users using limited caching resources. As a result, the serving time can be reduced.

REMARK 1. The obtained caching solution always achieves a higher SDP than the ground station most popular and uniform caching strategies.

\section{Numerical results}

THE values for key parameters are presented in Table I for illustrative purpose [26]. As more than $100 \mathrm{~dB}$ of self-interference (SI) cancellation level is required for the
FD system to achieve similar signal-to-noise-ratio-plusinterference as that of the HD system [27], SI cancellation parameter $\beta$ is chosen to be $10^{-4}$ to achieve SI cancellation level about $110 \mathrm{~dB}$ to $120 \mathrm{~dB}$ in our FD system setting. The fading states for the satellite-terrestrial links are defined by $\left\{m_{1}, b_{1}, \Omega_{1}\right\}=\{5,0.251,0.279\}$ [20] and $\left\{m_{2}, b_{2}, \Omega_{2}\right\}=\{4,0.126,0.835\}$ [21] when approaching average shadowing. End-users are randomly distributed inside a circle with radius $10 \mathrm{~km}$ and center at $G_{s}$.

TABLE I: Parameters Used for Numerical Results

\begin{tabular}{|c|c|c|c|}
\hline Parameter & Value & Parameter & Value \\
\hline $\mathrm{N}$ & 1000 files & $F$ & $100 \mathrm{Mb}$ \\
$B_{G} / B_{S} / B_{G_{s}}$ & $2 / 10 / 2 \mathrm{GHz}$ & $P_{S} / P_{G} / P_{G_{s}}$ & $10 / 30 / 2 \mathrm{~W}$ \\
$\sigma^{2}$ & $-120 \mathrm{dBm} / \mathrm{Hz}$ & $d_{S} / d_{G}$ & $35,786 \mathrm{~km}$ \\
$\alpha$ & 0.8 & $\alpha_{s} / \alpha_{g}$ & $2 / 3$ \\
$m_{1} / b_{1} / \Omega_{1}$ & $5 / 0.251 / 0.279$ & $m_{2} / b_{2} / \Omega_{2}$ & $4 / 0.126 / 0.835$ \\
$\xi_{i}$ & 0.35 & $P_{c, i} / P_{c, i}^{\prime}$ & $2.5 / 3 \mathrm{~W}$ \\
\hline \multicolumn{4}{|r}{} \\
\hline
\end{tabular}

\section{A. FD versus HD: Effect of SI Cancellation Parameter $\beta$}

We first investigate the average SDP when $\beta$ varies. Consider $C_{1}=50 G b, C_{2}=10 G b$, and $K=20$ users under uniform caching policy.

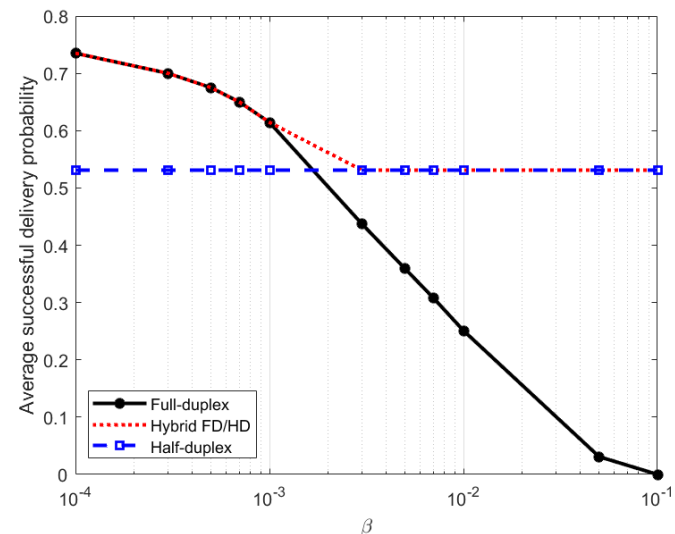

Fig. 7: Effect of the SIC parameter on average SDP.

With the HD scheme as a reference, the effect of $\beta$ on the FD scheme SDP is displayed in Fig. 7. As $\beta$ increases (i.e., worse self-interference cancellation in the FD system), the SDP decreases and starts getting worse than that of HD transmission. The FD scheme achieves the same SDP as that of HD with $\beta=1.7 \times 10^{-3}$. The self-interference cancellation in the FD system in this case is $103 \mathrm{~dB}$ to $107 \mathrm{~dB}$ which is almost the recommended value in [27]. The SDP of FD is $50 \%$ worse than that of HD with $\beta=10^{-1}$ where self-interference cancellation is $85 \mathrm{~dB}$ to $90 \mathrm{~dB}$. We also present numerical result for hybrid FD/HD transmission. In this hybrid scheme, the channel realizations are generated, then the delivery 
time of each file is the minimum delivery time between the FD and HD modes. In practical applications, once knowing the channel condition, devices in the system can dynamically switch between the FD and HD modes for better performance.

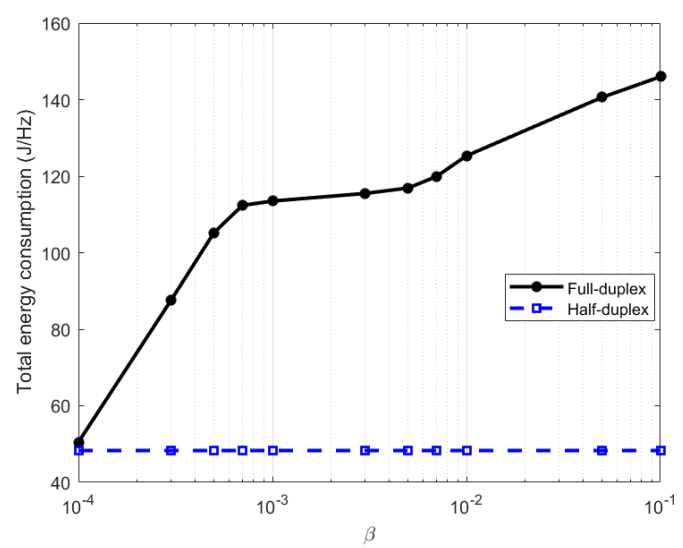

Fig. 8: Effect of the SIC parameter on energy consumption.

Fig. 8 shows the system total energy consumption when $\beta$ varies. With $\beta=10^{-4}$, which indicates to good SI cancellation, the two systems consume almost the same amount of energy but the FD system achieves $38 \%$ higher in SDP. When $\beta$ increases, the energy consumption in FD also increases and the SDP decreases. This amount of energy accounts for the SIC process and the energy for transmitting unsuccessful files.

\section{B. Two-tier versus Single-tier Caching}

The single-tier cache-enabled system in this section is adopted from STN system models in [8]-[10], where content caches are placed at ground stations only. The caching policies for the single-tier cache system correspond to those at the ground station in the two-tier cache system. For a fair comparison between the two-tier and single-tier caching systems, we set up both systems with the same total cache capacity of $80 \mathrm{~Gb}$ serving 20 users. The average SDPs of the uniform and ground station most popular caching policies are shown in Fig. 9.

Under the uniform caching policy, $N_{1} / N$ varies and $N_{2} / N$ is set to be 0.2 in the two-tier caching (meaning the satellite cache capacity is $20 \mathrm{~Gb}$ ). The result is shown in Fig. 9 with the two-tier scheme outperforming its singletier counterpart. At best performance with $N_{1} / N=0.7$, the achieved SDP in the two-tier scheme is $25 \%$ higher than that of the single-tier scheme. Under the ground station most popular caching policy, the number of most popular file cached $N_{1}$ varies; $N_{2}$ is fixed at 200 files in the two-tier scheme. The two-tier scheme gives approximately $40 \%$ SDP higher than that of the single-tier scheme in best performance scenarios with the ground station caching 600 most popular files. The single-tier system only outperforms the two-tier system when the

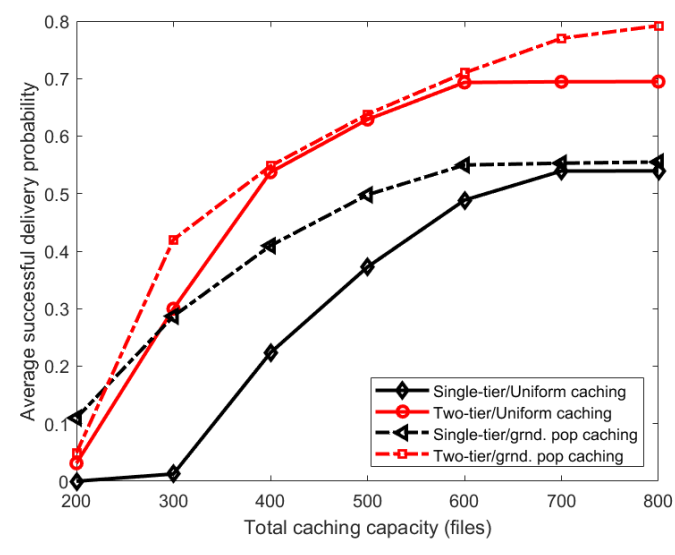

Fig. 9: Total caching capacity comparison.

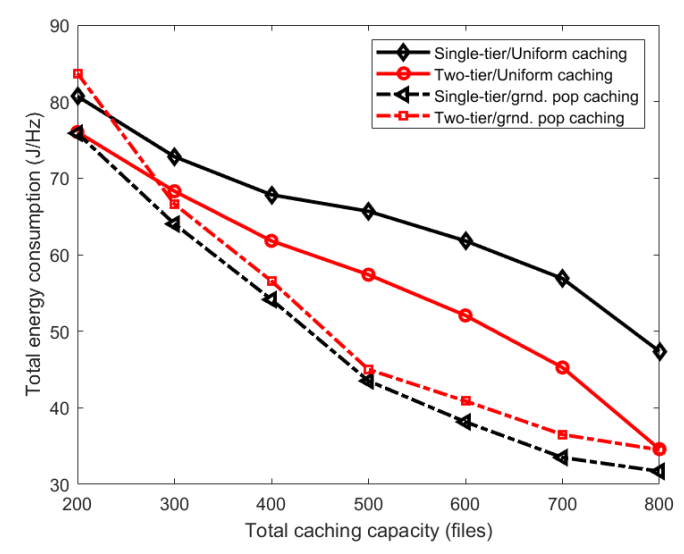

Fig. 10: Total energy consumption comparison.

total caching capacity is 200 files under uniform caching policy. In this case, we are comparing single-tier system with content cached solely at ground station and two-tier system with content cached at satellite only. The corresponding energy consumption is shown in Fig. 10. With more files cached, both systems consume less energy. Under ground station most popular caching policy, both systems achieve higher SDP while consume less energy than under uniform caching policy.

\section{FastForward versus without FastForward Relaying}

To compare the performance of the FD protocols, the system is set up with varying values for $C_{1}$ and $C_{2}$ of $20 \mathrm{~Gb}$ serving 20 users. Full-duplex with and without the FastForward relaying protocols are examined under the uniform and ground station most popular caching policies. File popularity is set to be $\alpha$ of 0.8 and $\beta$ of $10^{-4}$ for the FD mode. As shown in Fig. 11, the system with the FastForward relaying protocol achieves a higher SDP. This result is expected since the delivery time using the FastForward protocol is shorter than that without using FastForward relaying. 


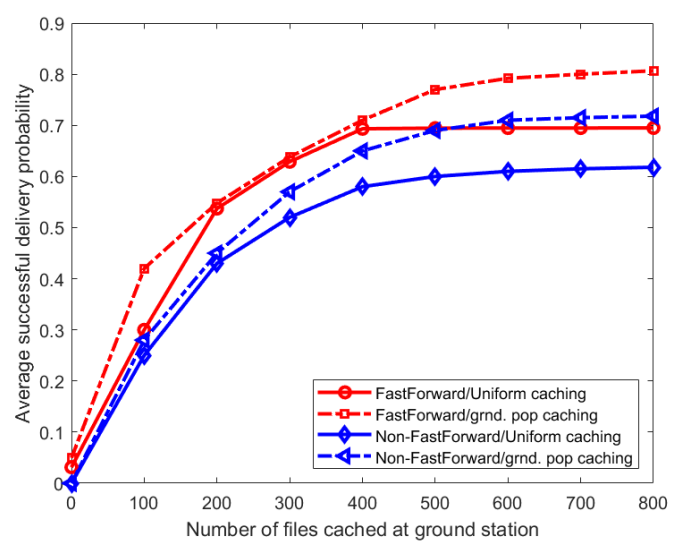

Fig. 11: Average SDP under FD with and without the FastForward relaying protocols.

\section{Network Capacity}

To examine the serving capability, the system is set up with the two layers' caches $C_{1}$ of $50 \mathrm{~Gb}$ and $C_{2}$ of $20 \mathrm{~Gb}$. The number of users $K$ varies while other parameters are listed in Table I. Fig. 12 shows the number of supportable users by the two-tier system under two caching policies. A smaller average SDP under uniform caching is achieved when supporting a small number of users; however, it surpasses the SDP of the ground station most popular caching scheme with a larger number of users. The acceptable SDP value depends on practical applications. In this case, with SDP of $50 \%$, the results follow a common understanding that the uniform caching scheme is not as effective as the ground station most popular caching scheme.

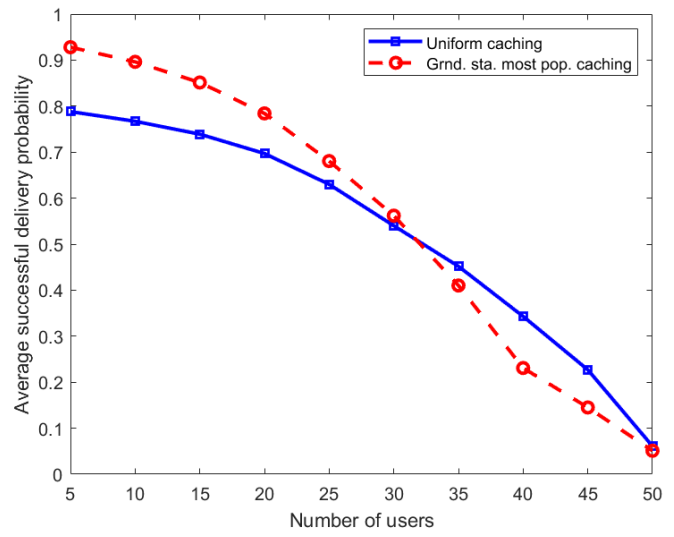

Fig. 12: Number of supportable users under different caching policies.

\section{E. Caching Capacity}

In this section, we analyze the two-tier caching capacity when serving 20 users. The system is configured using the parameters specified in Table I. The cache capacities of the two layers are shown in Fig. 13. As expected, the ground station most popular caching policy outperforms uniform caching. The more cache capacity is allocated to the ground station, the higher the SDPs are attainable; and the more content is cached at the satellite, the more the SDPs improve. Under uniform caching, the highest SDP is achieved at $70 \%$, while it is $80 \%$ under ground station most popular caching.

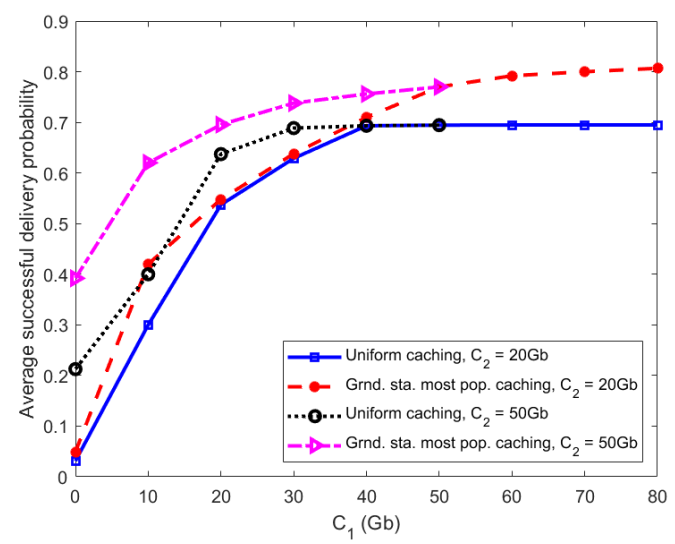

Fig. 13: Effect of caching capacity on the average SDP.

\section{F. Effect of File Popularity}

In this subsection, to examine the effect of the file popularity on the SDP under the ground station and satellite most popular caching policies, the system is configured with two tier caches $C_{1}$ and $C_{2}$ of $50 \mathrm{~Gb}$ and $20 \mathrm{~Gb}$, respectively, and serves 20 ground users. Through varying Zipf skewness factor $\alpha$, the resulting SDP is plotted in Fig. 14. Higher SDPs are achieved for both most popular caching schemes with an increase in the Zipf skewness factor. The results also match Proposition 1. This is because having one ground station in the system, the ground station most popular caching policy always gives better SDP results than under satellite most popular caching.

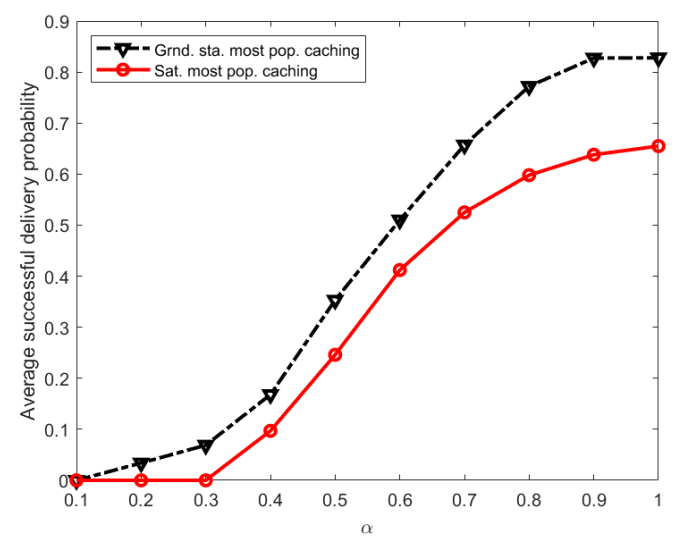

Fig. 14: Effect of file popularity on the average SDP. 


\section{G. Effect of the File Size}

To investigate the effect of the file size on SDP, we vary the sizes of $N$ files at the gateway following a uniform distribution with a mean of $100 \mathrm{Mb}$ and a standard deviation of $50 \mathrm{Mb}$. Two caching schemes are set up as follows: (i) $C_{1}$ is prefetched with the largest files and then $C_{2}$ is filled with the largest files among the remained files; (ii) $C_{2}$ is prefetched with the largest files and then $C_{1}$ is filled with the largest files among the remained files. The results are shown in Fig. 15. The average SDP is better when the largest files are cached at the ground station, resulting in a gain of approximately $10 \%$ to $20 \%$ in SDP.

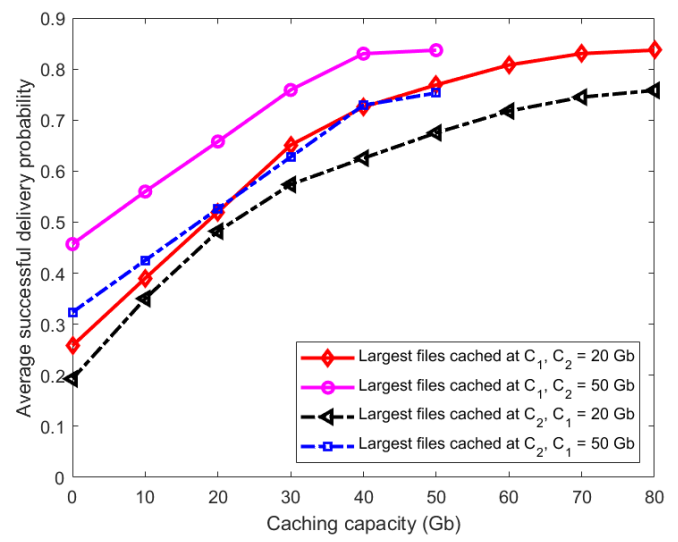

Fig. 15: Effect of the file size on the average SDP.

\section{H. Generalized Caching Solutions}

To investigate the performances of the generalized caching policies, we find the solutions when the caching capacity at satellite is fixed at $20 \mathrm{~Gb}$ and at ground station varies up to $80 \mathrm{~Gb}$. The performances are illustrated in Fig. 16. As can be seen from the figure, generalized caching policies yield higher SDPs than the naive uniform and ground station most popular caching policies. The solutions for each case of $C_{1}$ is detailed in Table II.

TABLE II: Solutions of The Generalized Caching Policies.

\begin{tabular}{|c|c|c|c|c|c|c|c|c|}
\hline$C_{1}$ & \multicolumn{4}{|c|}{ Generalized uniform } & \multicolumn{4}{|c|}{ Gen. grnd. sta. most pop. } \\
\cline { 2 - 9 }$(G b)$ & $N_{1}$ & $\bar{\mu}_{1}$ & $N_{2}$ & $\bar{\mu}_{2}$ & $N_{1}$ & $\bar{\mu}_{1}$ & $N_{2}$ & $\bar{\mu}_{2}$ \\
\hline 10 & 250 & 0.40 & 400 & 0.50 & 220 & 0.45 & 370 & 0.54 \\
20 & 350 & 0.57 & 500 & 0.40 & 300 & 0.67 & 370 & 0.54 \\
30 & 450 & 0.66 & 650 & 0.30 & 500 & 0.60 & 330 & 0.60 \\
40 & 650 & 0.61 & 650 & 0.30 & 570 & 0.70 & 300 & 0.66 \\
50 & 700 & 0.71 & 800 & 0.25 & 600 & 0.83 & 280 & 0.71 \\
60 & 800 & 0.75 & 900 & 0.22 & 600 & 1.00 & 200 & 1.00 \\
70 & 910 & 0.76 & 900 & 0.22 & 700 & 1.00 & 200 & 1.00 \\
80 & 950 & 0.84 & 1000 & 0.15 & 800 & 1.00 & 200 & 1.00 \\
\hline
\end{tabular}

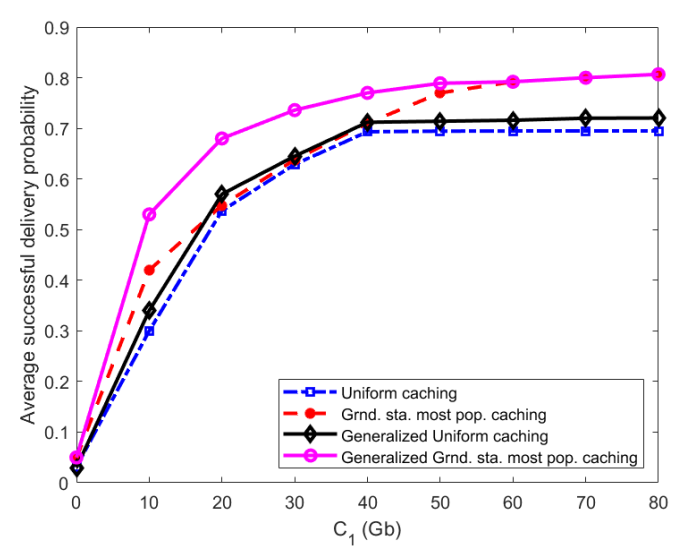

Fig. 16: Average SDP under the generalized caching policies.

\section{Optimal Caching Solution}

Under generic caching policy, the original optimization problem in (13) could be solved in case of a small number of files $N$. For the purpose of illustration, we compute the optimal solution for the problem in (13) in the case of $N=20$ in Fig. 17. The caching capacity at satellite is fixed at $1 \mathrm{~Gb}$ and at ground station varies up to $4 \mathrm{~Gb}$. The optimal solution (generic caching) of the original problem in (13) is found using exhaustive search. For the optimal solution, with one constraint on the capacity of the two cache tiers $C_{1}$ and $C_{2}$, we generate feasible caching solutions by computing its SDP values using (9), and then determining the best one.

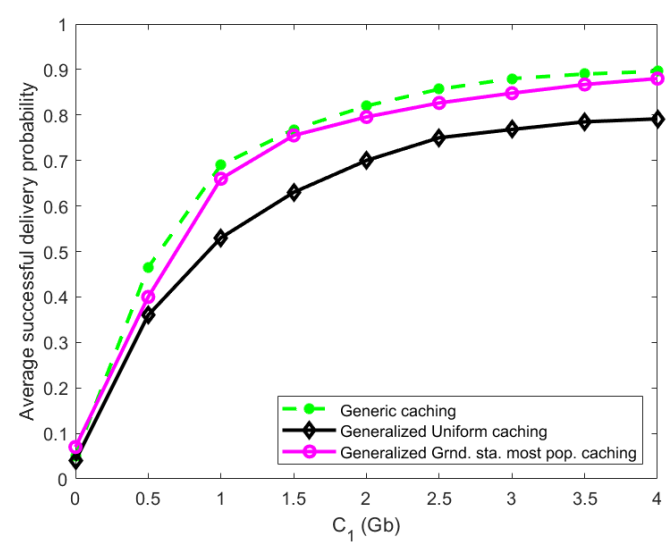

Fig. 17: Optimal caching solution.

As expected, the optimal solution under generic caching gives the best SDP comparing to the sub-optimal solution under the two generalized caching policies. The results show that the gaps between the optimal and suboptimal solutions are quite negligible, demonstrating the effectiveness of the caching policies under consideration at significantly reduced computational complexity. 


\section{Conclusion}

IN this paper, we studied the SDP of a two-tier cache enabled satellite IoT system with full-duplex communications. A thorough analysis for the SDP under the FD [14] mode with the FastForward relaying protocol was carried out under different caching policies. Cache placement was also designed with the goal of maximizing the [15] average SDP. Using the FD mode without the FastForward protocol, HD mode and single-tier cache system as benchmarks, numerical results were presented when investigating the system performance in terms of SDP, maximum number of supportable users, caching capacity in each tier, and the effect of file popularity on the system performance under different caching policies. For a better understanding on parameters that affect the proposed system performance, a special case with a variety of file sizes was also investigated.

\section{REFERENCES}

[1] S. K. Routray and H. M. Hussein, "Satellite based iot networks for [19] emerging applications," arXiv:1904.00520, Mar. 2019. [Online]. Available: https://arxiv.org/abs/1904.00520

[2] A. G. et al., "Architectures and key technical challenges for 5g systems incorporating satellites," IEEE Transactions on Vehicular [20] Technology, vol. 68, no. 3, pp. 2624-2639, Mar. 2019.

[3] Sat5G, "Satellite and terrestrial network for 5g," Available at https://www.sat5g-project.eu/wp-content/uploads/2019/ 04/Sat5g-project-document-2019-v31.pdf (2021/08/15).

[4] FCC Report, "Starlink fcc application," Available at https://fcc. report/IBFS/SAT-MOD-20181108-00083 (2020-07-21).

[5] Y. Li, Y. Wang, P. Yuan, Q. Zhang, and Z. Yang, "Popularityaware back-tracing partition cooperative cache distribution for space-terrestrial integrated networks," IET Communications, vol. 13, no. 17, pp. 2786-2796, Oct. 2019.

[6] Z. Yang, Y. Li, P. Yuan, and Q. Zhang, "Tcsc: A novel file dis- ${ }^{[23]}$ tribution strategy in integrated leo satellite-terrestrial networks," IEEE Transactions on Vehicular Technology, vol. 69, no. 5, pp. 5426-5441, May 2020.

[7] J. Li, K. Xue, D. S. L. Wei, J. Liu, and Y. Zhang, "Energy efficiency and traffic offloading optimization in integrated satellite terrestrial radio access networks," IEEE Transactions on Wireless Communications, vol. 19, no. 4, pp. 2367-2381, Apr. 2020.

[8] K. An, Y. Li, X. Yan, and T. Liang, "On the performance of cache-enabled hybrid satellite-terrestrial relay networks," IEEE Wireless Communications Letters, vol. 8, no. 5, pp. 1506-1509, Jun. 2019.

[9] A. Kalantari, M. Fittipaldi, S. Chatzinotas, T. X. Vu, and B. Ottersten, "Cache-assisted hybrid satellite-terrestrial backhauling for $5 \mathrm{~g}$ cellular networks," in 2017 IEEE Global Communications Conference (GLOBECOM), Singapore, 2017, pp. 1-6.

[10] T. Vu, Y. Poirier, S. Chatzinotas, and N. Maturo, "Modeling and implementation of $5 \mathrm{~g}$ edge caching over satellite," International Journal of Satellite Communications and Networking, vol. 38, no. 5, pp. 395-406, Mar. 2020.

[11] H. Wu, J. Li, H. Lu, and P. Hong, "A two-layer caching model for content delivery services in satellite-terrestrial networks," in 2016 IEEE Global Communications Conference (GLOBECOM), DC, USA, 2016, pp. 1-6.

[12] L. Liu, J. Zhang, X. Zhang, P. Wang, Y. Wang, and L. Ouyang, "Design and analysis of cooperative multicast-unicast transmission scheme in hybrid satellite-terrestrial networks," in 2018 IEEE International Conference on Communication Systems (ICCS), Wuxi, China, 2018, pp. 309-314.
G. S. Paschos, G. Iosifidis, M. Tao, D. Towsley, and G. Caire, "The role of caching in future communication systems and networks," IEEE J. Sel. Areas Commun., vol. 36, no. 6, pp. 1111-1125, Sept. 2018.

Z. Zhang, W. Zhang, and F. Tseng, "Satellite mobile edge computing: Improving qos of high-speed satellite-terrestrial networks using edge computing techniques," IEEE Network, vol. 33, no. 1, pp. 70-76, Jan. 2019.

Q. Ngo, K. Phan, W. Xiang, A. Mahmood, and J. Slay, "Two-tier cache-aided full-duplex content delivery in satellite-terrestrial networks," in 10th International Conference on Computational Data and Social Networks (CSoNet 2021), Montreal, Canada, 2021.

T. X. Vu, L. Lei, S. Chatzinotas, B. Ottersten, and A. V. Trinh, "On the successful delivery probability of full-duplex-enabled mobile edge caching," IEEE Communications Letters, vol. 23, no. 6, pp. 1016-1020, Jun. 2019.

L. Breslau, P. Cao, L. Fan, G. Phillips, and S. Shenker, "Web caching and Zipf-like distributions: Evidence and implications," in IEEE Conf. Comput. Commun. Soc. (INFOCOM), NY, USA, 1999 , pp. $126-134$.

[18] A. Abdi, W. C. Lau, M. Alouini, and M. Kaveh, "A new simple model for land mobile satellite channels: first- and second-order statistics," IEEE Trans. Wireless Commun., vol. 2, no. 3, pp. 519-528, May 2003.

M. R. Bhatnagar and A. M.K., "Performance analysis of af based hybrid satellite-terrestrial cooperative network over generalized fading channels," IEEE Communications Letters, vol. 17, no. 10, pp. 1912-1915, Sept. 2013.

N. I. Miridakis, D. D. Vergados, and A. Michalas, "Dual-hop communication over a satellite relay and shadowed rician channels," IEEE Trans. Veh. Technol., vol. 64, no. 9, pp. 4031-4040, Sept. 2015.

[21] K. Guo, K. An, B. Zhang, Y. Huang, D. Guo, G. Zheng, and S. Chatzinotas, "On the performance of the uplink satellite multiterrestrial relay networks with hardware impairments and interference," IEEE Systems Journal, vol. 13, no. 3, pp. 2297 2308, Sept. 2019.

I. S. Gradshteyn and I. M. Ryzhik, Tables of Integrals, Series and Products, vol. 6th ed., 2000.

S. Vural, P. Navaratnam, N. Wang, C. Wang, L. Dong, and R. Tafazolli, "In-network caching of internet-of-things data," in 2014 IEEE International Conference on Communications (ICC), Sydney, Australia, 2014, pp. 3185-3190.

K. T. Phan and T. Le-Ngoc, "Power allocation for buffer-aided fullduplex relaying with imperfect self-interference cancelation and statistical delay constraint," IEEE Access, vol. 4, pp. 3961-3974, Jul. 2016.

25] B. Dinesh and K. Sachin, "Fastforward: Fast and constructive full duplex relays," in Proceedings of the 2014 ACM Conference on SIGCOMM (SIGCOMM '14), IL, USA, 2014, p. 199-210.

HughesNet, "Echostar xix satellite," Available at https://www. hughesnet.com/whats-new/satellite-launch (2020-07-21).

H. Li, J. Van Kerrebrouck, O. Caytan, H. Rogier, J. Bauwelinck, P. Demeester, and G. Torfs, "Self-interference cancellation enabling high-throughput short-reach wireless full-duplex communication," IEEE Trans. Wireless Commun., vol. 17, no. 10, pp. 6475-6486, Aug. 2018. 


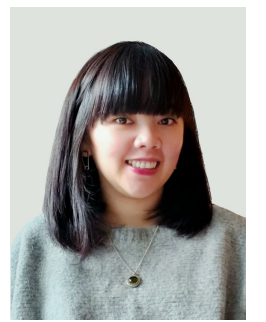

Quynh T. Ngo is currently a $\mathrm{PhD}$ candidate at the Department of Computer Science and Information Technology, La Trobe University, Melbourne, Australia. She received the B.Sc. in Electrical Engineering (Magna Cum Laude) from the California State University Los Angeles, CA, USA, in 2013, and the M.Sc. in Telecommunications from the Vietnam National University - University of Sciences, HCM, Vietnam, in 2016. Her research interests are medium access control for vehicular ad-hoc networks, edge caching and physical layer security for satellite-IoT. She has been awarded the Jet Propulsion Laboratory Undergraduate Scholar (JPLUS), the visiting research award funded by the Norwegian - Vietnamese Industrial \& Infrastructure Safety Consortium, the La Trobe University Post Graduate Research Scholarship, and the Net Zero Scholarship.

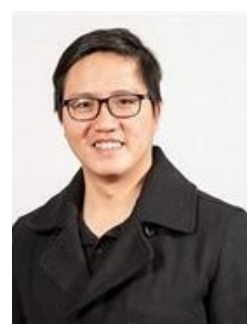

Khoa T. Phan (Member, IEEE) received the B.Eng. degree in telecommunications (First Class Hons.) from the University of New South Wales (UNSW), Sydney, NSW, Australia, in 2006, the M.Sc. degree in electrical engineering from the University of Alberta, Edmonton, $\mathrm{AB}$ Canada, in 2008, and California Institute of Technology (Caltech), Pasadena, CA, USA, in 2009, respectively, and the Ph.D. degree in electrical engineering from McGill University, Montreal, QC, Canada in 2017. He is currently a Senior Lecturer and Australia Research Council (ARC) Discovery Early Career Researcher Award (DECRA) Fellow with the Department of Computer Science and Information Technology, La Trobe University, Victoria, Australia. $\mathrm{He}$ was a Researcher with the Electrical Engineering Department, University of California, Los Angeles (UCLA), CA, USA, from 2009 to 2011. From 2017 to 2018, he was a Research Fellow with the Electrical and Computer Systems Engineering (ECSE) Department, Monash University, Melbourne, Australia. His current research interests are broadly design, control, optimization, and operation of 5G mobile communications networks with applications in the Internet of Things (IoT), satellite communications, machine-type communications (MTC), smart grids, and cloud computing. Dr. Phan has been awarded several prestigious fellowships including the Australian Development Scholarship (UNSW), the Alberta Ingenuity Fund Student Fellowship and the iCORE Graduate Student Award (Alberta), the Atwood Fellowship (Caltech), the McGill Doctoral Engineering Award, the SRTelecom Doctoral Award (McGill), and ARC DECRA (La Trobe).

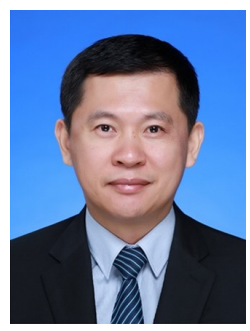

Wei Xiang (Senior Member, IEEE) Professor Wei Xiang is Cisco Research Chair of AI and IoT and Director of the Cisco-La Trobe Centre for AI and IoT at La Trobe University. Previously, he was Foundation Chair and Head of Discipline of IoT Engineering at James Cook University, Cairns, Australia. Due to his instrumental leadership in establishing Australia's first accredited Internet of Things Engineering degree program, he was inducted into Pearcy Foundation's Hall of Fame in October 2018. He is an elected Fellow of the IET in UK and Engineers Australia. He received the TNQ Innovation Award in 2016, and Pearcey Entrepreneurship Award in 2017, and Engineers Australia Cairns Engineer of the Year in 2017. He was a co-recipient of four Best Paper Awards at WiSATS'2019, WCSP'2015, IEEE WCNC'2011, and ICWMC'2009. He has been awarded several prestigious fellowship titles. He was named a Queensland International Fellow (2010-2011) by the Queensland Government of Australia, an Endeavour Research Fellow (2012-2013) by the Commonwealth Government of Australia, a Smart Futures Fellow (2012-2015) by the Queensland Government of Australia, and a JSPS Invitational Fellow jointly by the Australian Academy of Science and Japanese Society for Promotion of Science (2014-2015). He was the Vice Chair of the IEEE Northern Australia Section from 2016-2020. He was an Editor for IEEE Communications Letters (2015-2017), and is currently an Associate Editor for IEEE Communications Surveys \& Tutorials, IEEE Internet of Things Journal, IEEE Access, and Nature journal of Scientific Reports. He has published over 250 peer-reviewed papers including 3 books and 200 journal articles. He has severed in a large number of international conferences in the capacity of General Co-Chair, TPC CoChair, Symposium Chair, etc. His research interest includes the Internet of Things, wireless communications, machine learning for IoT data analytics, and computer vision.

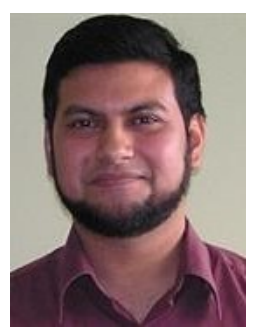

Abdun Mahmood (Senior Member, IEEE) Dr. Abdun Mahmood received his $\mathrm{PhD}$ from the University of Melbourne, Australia, in 2008 the MSc (Research) degree in computer science and the B.Sc. degree in applied physics and electronics from the University of Dhaka, Bangladesh, in 1999 and 1997, respectively. Dr. Mahmood had an academic career in University since 2000, working at University of Dhaka, RMIT University, UNSW Canberra and currently in La Trobe University as an Associate Professor (Reader).

Dr. Mahmood leads a group of researchers focusing on Machine Learning and Cybersecurity including Anomaly Detection in Smart Grid, SCADA security, Memory Forensics, and False Data Injection. Dr. Mahmood has been successful to attract over a $\$ 1 \mathrm{M}+$ in grant funding as a CI, including two ARC Linkage Projects.

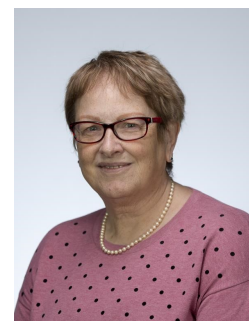

Jill Slay (Member, IEEE) Professor Jill Slay is the University of South Australia SmartSa CRC Professorial Chair in Cybersecurity. Her work focuses on the context of developing the national technical agenda in satellite cybersecurity and resilience with the Australian Defence Science and Technology Group, Defence and Defence Industry. She is the Chair of the Australian Women in Security Network (900 members) and a Director of the International Information Systems Security Certification Consortium for 2021-2023. Previous appointments have included Optus Chair in Cybersecurity at La Trobe University, and Founding Director of the Australian Centre for Cyber Security at the Australian Defence Force Academy. She has established an international research reputation in cyber security (particularly Digital Forensics, Cyber Intelligence and Cyberwarfare) and has worked in collaboration with the Australian Federal and State governments and with many industry partners. She has published more than 140 outputs in information assurance, critical infrastructure protection, security and forensic computing and completed the supervision of $20 \mathrm{PhDs}$ and many master's and Honour's theses. She was made a Member of the Order of Australia (AM) for service to the information technology industry through contributions in the areas of forensic computer science, security, protection of infrastructure and cyber-terrorism. 